\title{
Correction to: Present and the Future of Space Internet: The Space Generation Perspective by Bacsardi L, Birkeland R, Hornig A, Morrison B, Shar M, and Tsodikovich Y. New Space 2017;5:257-267. DOI: $10.1089 /$ space.2017.0018
}

In the December 2017 issue of New Space (vol. 5, no. 4; 257-267) the article entitled "Present and the Future of Space Internet:

The Space Generation Perspective" by Bacsardi et al. requires correction.

The author list was intended to be published alphabetically, however, two authors were inadvertently transposed. The author list originally appeared as:

Laszlo Bacsardi, ${ }^{1}$ Roger Birkeland, ${ }^{2}$ Andreas Hornig, ${ }^{3}$

Mansoor Shar, ${ }^{4}$ Brandon Morrison, ${ }^{5}$ and Yevgeny Tsodikovich ${ }^{6}$

${ }^{1}$ University of Sopron, Sopron, Hungary.

${ }^{2}$ Norwegian University of Science and Technology, Trondheim, Norway.

${ }^{3}$ University of Stuttgart, Stuttgart, Germany.

${ }^{4}$ International Space University, Strasbourg, France.

${ }^{5}$ Durham University, Durham, United Kingdom.

${ }^{6}$ Tel Aviv University, Tel Aviv, Israel.

The author list should have been:

Laszlo Bacsardi, ${ }^{1}$ Roger Birkeland, ${ }^{2}$ Andreas Hornig, ${ }^{3}$

Brandon Morrison, ${ }^{4}$ Mansoor Shar, ${ }^{5}$ and Yevgeny Tsodikovich ${ }^{6}$

${ }^{1}$ University of Sopron, Sopron, Hungary.

${ }^{2}$ Norwegian University of Science and Technology, Trondheim, Norway.

${ }^{3}$ University of Stuttgart, Stuttgart, Germany.

${ }^{4}$ Durham University, Durham, United Kingdom.

${ }^{5}$ International Space University, Strasbourg, France.

${ }^{6}$ Tel Aviv University, Tel Aviv, Israel.

The online version of the article has been corrected to reflect this. 